\title{
Walnut Consumption Induces Tissue-Specific Omega-6/Omega-3 Decrease in High-Fructose-Fed Wistar Rats
}

\author{
Manja M. Zec, ${ }^{*}$, Irena Krga, $^{\S}$ Marija Takić, Jasmina Debeljak-Martačić, Goran Korićanac, \\ Slavica Ranković, Tamara Popović, Marija Pantelić, and Maria Glibetic
}

Cite This: ACS Omega 2020, 5, 28136-28145

Read Online

ACCESS I

山ll Metrics \& More

Article Recommendations

Supporting Information

ABSTRACT: Increased dietary, blood, and tissue n-6/n-3 fatty acid ratios are associated with obesity and metabolic syndrome. Due to Westernized dietary patterns, the increasing n-6/n-3 ratio is of growing concern worldwide, and dietary strategies aimed at its lowering are of public health importance. Walnuts are rich in dietary fats, and their consumption promotes cardiometabolic health. This study aimed to examine the effect of 6 -week walnut consumption on tissue-specific n-6/ n-3 ratio and fatty acid metabolic conversion in fructose-fed rats with a cluster of metabolic disorders. Male Wistar rats were fed a standard diet with or without $10 \%$ fructose in drinking water for 9 weeks. Diets of half of the animals were then

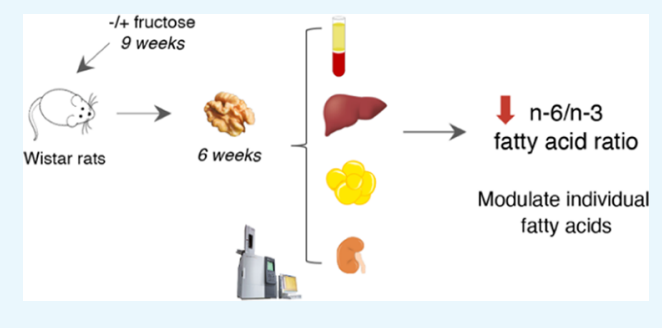
supplemented with walnuts $(2.4 \mathrm{~g} /$ day $)$ for 6 weeks, upon which fatty acid profiles were determined in plasma, liver, adipose tissue, and kidney total lipids. Results showed that walnuts induced significant decreases in the $n-6 / n-3$ content of total lipid pool in plasma and examined tissues, irrespective of metabolic burden. Walnut intervention decreased plasma and liver palmitoleic/palmitic, arachidonic/linoleic, and docosahexaenoic/ $\alpha$-linolenic acid ratios. It also modulated individual fatty acid levels by reducing arachidonic and palmitic acid and increasing $\alpha$-linolenic, eicosapentaenoic, and docosapentaenoic acid in plasma and most tissues. Our study demonstrated that 6-week consumption of walnuts favorably modulated n-6/n-3 plasma and tissue ratio in male Wistar rats regardless of high-fructose feeding, underscoring the promising potential of walnuts in both prevention and treatment of the metabolic syndrome.

\section{INTRODUCTION}

Metabolic syndrome (MetS) is a cluster of metabolic disturbances, including obesity, dyslipidemia, elevated blood pressure, and impaired glucose and insulin metabolism, associated with increased cardiovascular risk. ${ }^{1}$ Dietary fat intake $^{2}$ and plasma lipid profiles as seen in fatty acid distribution $^{3}$ are linked with the components of MetS in subjects at high cardiovascular risk., ${ }^{2,3}$ Essential fatty acids, including linoleic acid (LA, C18:2 n-6) and $\alpha$-linolenic acid ( $\alpha$ LNA, C18:3 n-3), are important as humans are not capable of synthesizing them and are thus taken exclusively from the diet. The LA and $\alpha$ LNA are further endogenously metabolized to long-chain unsaturated omega-6 (n-6) and omega-3 (n-3) products, using the desaturase and elongase enzymes, the latter also implicated in MetS development. ${ }^{3}$ Westernized dietary patterns have induced increase in n-6 in place of n-3 fatty acid intake worldwide, and dietary strategies aimed at the intake optimization are of public health importance. ${ }^{4}$ Moreover, the ratio between total $n-6$ and $n-3$ content $(n-6 / n-3)$ in diet, blood, and tissues has an important role in obesity and metabolic syndrome. $^{5}$

Nuts are rich in dietary fats, and their consumption is unambiguously linked with various health-promoting effects. ${ }^{6}$ Among other nuts, walnuts are particularly rich in $\alpha \mathrm{LNA},{ }^{7}$ which is endogenously converted to long-chain products: eicosapen- taenoic (EPA, C20:5 n-3) and docosahexaenoic fatty acid (DHA, C22:6 n-3) ${ }^{7}$ with anti-inflammatory, endothelialmodulatory, triglyceride-lowering, and blood pressure-modulating effects. ${ }^{8}$ Thus, the intake of walnuts is of special relevance in countries with low marine food intake. ${ }^{9}$ Furthermore, high carbohydrate and fructose intake is linked with de novo lipogenesis (DNL) pathway, ${ }^{10}$ including the metabolism of palmitic acid (PA, C16:0), stearic acid (C18:0), and palmitoleic acid (POA, C16:1 n-7), ${ }^{11}$ and the POA/PA ratio is directly linked with MetS. ${ }^{3}$ Cohort studies confirmed that regular consumption of walnuts is a step ahead of other nut types with regard to cardiovascular disease incidence reduction, including coronary heart disease and stroke. ${ }^{12}$ A recent meta-analysis of randomized control trials showed that, among other nuts, only walnuts induced a significant improvement in flow-mediated dilatation. ${ }^{13}$ Furthermore, the PREDIMED study showed that replacing $30 \mathrm{~g}$ of nuts with an equivalent walnut portion might be beneficial toward lower mean body weight in subjects at

Received: August 6, 2020

Accepted: October 8, 2020

Published: October 21, 2020 


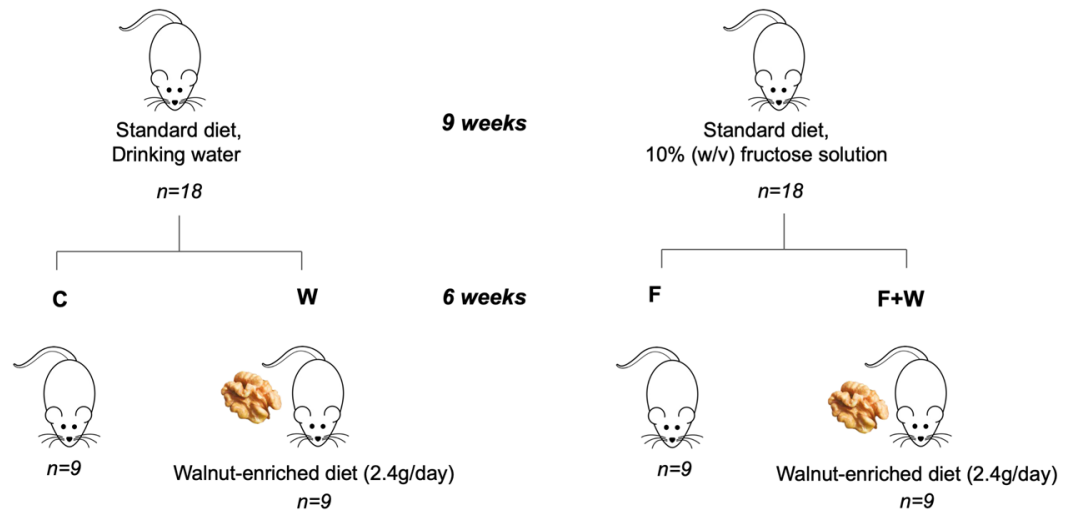

Figure 1. Study design. $\mathrm{C}$ - control, $\mathrm{W}$-walnuts, $\mathrm{F}$-fructose, $\mathrm{F}+\mathrm{W}$-fructose plus walnuts group.

A

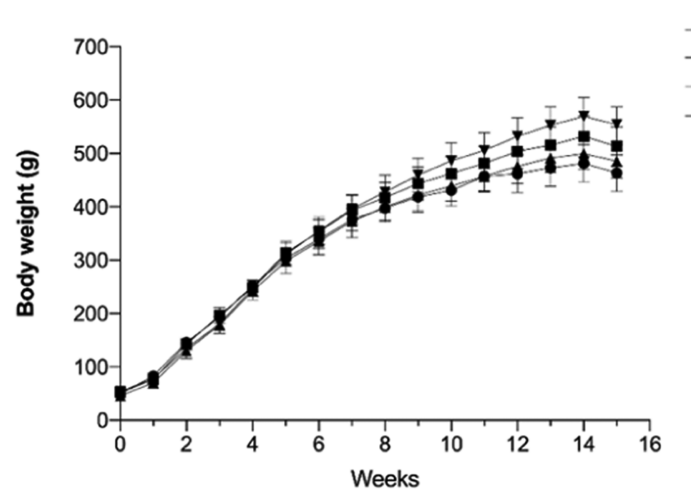

B

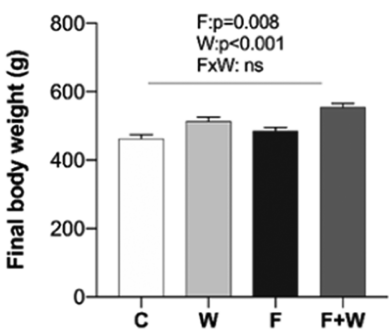

C

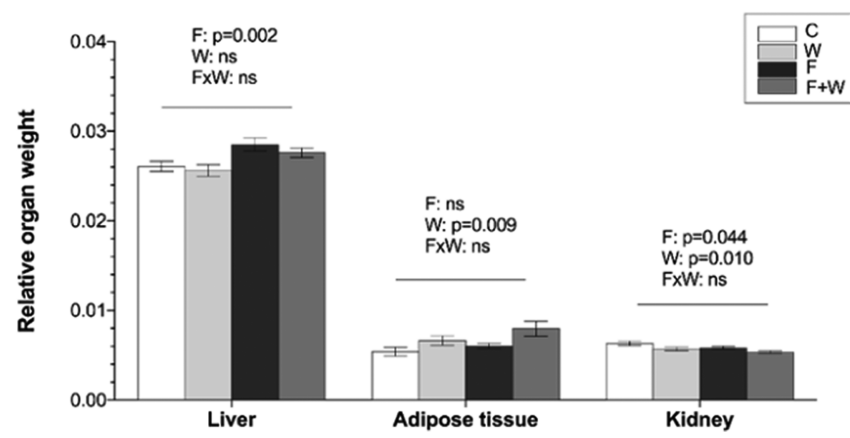

Figure 2. Effects of walnut consumption on body weight and relative organ weight. (A) Body weight changes, (B) final body weight, and (C) relative liver, epididymal adipose tissue, and kidney weight, in fructose-naive or fructose-fed male rats with or without dietary supplementation with walnuts for 6 weeks. Relative organ weight was calculated as organ weight/body weight. Data are analyzed by two-way analysis of variance (ANOVA), with fructose and walnut treatments as independent factors. Results are presented as mean \pm standard error of the mean $(\mathrm{SEM}), n=8-9$. C-control, Wwalnuts, $\mathrm{F}$-fructose, $\mathrm{F}+\mathrm{W}$-fructose plus walnuts.

cardiovascular risk. ${ }^{14}$ Hence, there is a continuing interest in the safe consumption of walnuts.

An increasing number of animal studies suggest diverse cardiometabolic effects of walnut consumption, including their capacity to improve lipid profiles; ${ }^{15}$ exert hepatoprotective, antioxidant, ${ }^{16}$ and anti-inflammatory effects; ${ }^{17}$ and modulate cell signaling pathways involved in the regulation of lipid metabolism. ${ }^{18-20}$ A study in Wistar rats showed that walnut oil consumption alleviated free fatty acid load imposed by the highcarbohydrate or high-fat diet, which was followed by downregulation of NF- $\kappa \mathrm{B}$-mediated pathway in the liver. ${ }^{21}$ Walnuts are rich in phytosterols, and phytosterol ester consumption in rats with nonalcoholic fatty liver disease is followed by decreased saturated/monounsaturated fatty acid load in serum, liver, and adipose tissue. ${ }^{22}$ However, animal studies investigating the effects of whole walnuts consumption on the tissue-specific fatty acid distribution in the presence of metabolic risk are lacking.

Walnuts are also rich in LA, and metabolic competition between LA and $\alpha$ LNA conversion due to bioavailability might lead to a differential n-6/n-3 tissue-specific ratio. ${ }^{23}$ A previous small-scale study in asymptomatic subjects indicated a decrease in the $n-6 / n-3$ ratio in plasma phospholipids upon 4-week walnut consumption, due to the higher EPA and $\alpha$ LNA plasma content. ${ }^{9}$ Therefore, this consumption study was designed to assess the effects of whole walnuts on omega-6/omega- 3 tissuespecific content in high-fructose-fed male Wistar rats with a cluster of metabolic disturbances. We then evaluated the effects on desaturation and elongation metabolic capacity, estimated 
Table 1. Biochemical Parameters in Wistar Rats ${ }^{a}$

\begin{tabular}{|c|c|c|c|c|c|c|c|}
\hline & \multicolumn{2}{|c|}{ fructose-naïe } & \multicolumn{2}{|c|}{ fructose-fed } & \multirow[b]{2}{*}{$F^{\mathrm{a}}$} & \multirow[b]{2}{*}{$\mathrm{W}^{\mathrm{b}}$} & \multirow[b]{2}{*}{$\mathrm{FxW}^{\mathrm{c}}$} \\
\hline & control & walnuts & fructose & fructose + walnuts & & & \\
\hline glucose $(\mathrm{mmol} / \mathrm{L})$ & $5.21 \pm 0.14$ & $5.20 \pm 0.13$ & $5.54 \pm 0.19$ & $5.30 \pm 0.14$ & 0.162 & 0.405 & 0.447 \\
\hline $\operatorname{ALT}(\mathrm{U} / \mathrm{L})$ & $50.81 \pm 2.88$ & $51.37 \pm 3.0$ & $46.74 \pm 4.99$ & $43.87 \pm 2.49$ & 0.106 & 0.741 & 0.625 \\
\hline $\mathrm{AST}(\mathrm{U} / \mathrm{L})$ & $115.80 \pm 7.72$ & $120.34 \pm 8.52$ & $128.47 \pm 16.7$ & $93.86 \pm 4.57$ & 0.511 & 0.158 & 0.069 \\
\hline TAG (mmol/L) & $0.85 \pm 0.06$ & $0.96 \pm 0.09$ & $1.02 \pm 0.09$ & $1.33 \pm 0.17$ & 0.019 & 0.060 & 0.349 \\
\hline uric acid $(\mu \mathrm{mol} / \mathrm{L})$ & $28.72 \pm 1.90$ & $28.42 \pm 1.03$ & $31.42 \pm 2.91$ & $24.59 \pm 1.40$ & 0.773 & 0.076 & 0.103 \\
\hline creatinine $(\mu \mathrm{mol} / \mathrm{L})$ & $29.70 \pm 0.87$ & $31.34 \pm 0.88$ & $27.14 \pm 0.85$ & $26.52 \pm 0.86$ & $<0.001$ & 0.559 & 0.199 \\
\hline $\mathrm{TC}(\mathrm{mmol} / \mathrm{L})$ & $2.29 \pm 0.10$ & $2.02 \pm 0.11$ & $2.55 \pm 0.18$ & $2.33 \pm 0.08$ & 0.031 & 0.056 & 0.821 \\
\hline LDL-c (mmol/L) & $0.39 \pm 0.04$ & $0.31 \pm 0.03$ & $0.45 \pm 0.06$ & $0.42 \pm 0.04$ & 0.050 & 0.230 & 0.576 \\
\hline HDL-c (mmol/L) & $1.91 \pm 0.07$ & $1.69 \pm 0.09$ & $2.17 \pm 0.14$ & $1.91 \pm 0.08$ & 0.020 & 0.019 & 0.821 \\
\hline TC/HDL-c & $1.20 \pm 0.02$ & $1.19 \pm 0.02$ & $1.17 \pm 0.01$ & $1.23 \pm 0.02$ & 0.930 & 0.196 & 0.104 \\
\hline LDL-c/HDL-c & $0.20 \pm 0.01$ & $0.18 \pm 0.02$ & $0.20 \pm 0.01$ & $0.22 \pm 0.01$ & 0.219 & 0.910 & 0.279 \\
\hline
\end{tabular}

${ }^{a}$ Data are presented as mean \pm SEM, $n=9$. ${ }^{a, b, c}$ Probabilities upon two-way ANOVA for fructose effect $(F)$, walnut effect (W), and interaction between fructose and walnuts (FxW), respectively, upon 6 weeks. ALT, alanine aminotransferase; AST, aspartate aminotransferase; HDL-c, highdensity lipoprotein cholesterol; LDL-c, low-density lipoprotein cholesterol; TAG, triglycerides; TC, total cholesterol.

Table 2. Effects of Walnut Feeding on the Tissue-Specific n-6/n-3 Ratio in Fructose-Fed and Control Male Wistar Rats ${ }^{a}$

\begin{tabular}{|c|c|c|c|c|c|c|c|}
\hline & \multicolumn{2}{|c|}{ fructose-naive } & \multicolumn{2}{|c|}{ fructose-fed } & \multirow[b]{2}{*}{$\mathrm{F}^{\mathrm{a}}$} & \multirow[b]{2}{*}{$\mathrm{W}^{\mathrm{b}}$} & \multirow[b]{2}{*}{$\mathrm{FxW}^{\mathrm{c}}$} \\
\hline & control & walnuts & fructose & fructose + walnuts & & & \\
\hline plasma & $16.73 \pm 1.58$ & $9.00 \pm 0.48$ & $14.86 \pm 1.30$ & $10.65 \pm 0.55$ & 0.919 & $<0.001$ & 0.112 \\
\hline liver & $8.19 \pm 0.31$ & $5.77 \pm 0.26$ & $8.35 \pm 0.48$ & $7.07 \pm 0.36$ & 0.055 & $<0.001$ & 0.129 \\
\hline adipose tissue & $22.13 \pm 1.02$ & $13.17 \pm 0.99$ & $22.04 \pm 1.54$ & $10.97 \pm 0.40$ & 0.290 & $<0.001$ & 0.329 \\
\hline kidney & $34.12 \pm 2.32$ & $21.72 \pm 1.57$ & $35.08 \pm 3.03$ & $26.35 \pm 1.95$ & 0.231 & $<0.001$ & 0.429 \\
\hline
\end{tabular}

${ }^{a}$ Data are presented as mean \pm SEM, $n=7-9$. ${ }^{\text {a, }, \mathrm{b}, \mathrm{c}}$ Probabilities upon two-way ANOVA for fructose effect (F), walnuts effect (W), and interaction between fructose and walnuts $(\mathrm{FxW})$, respectively, upon 6 weeks.

through the product-to-precursor ratio in omega-7, omega-3, and omega- 6 metabolic pathways. We further investigated the effects on biochemical parameters, as well as the tissue-specific distribution of individual fatty acids. To evaluate the potential of walnuts consumption in the established metabolic disorder, fructose was applied 9 weeks prior to the commencement of the walnut consumption and was continued during the walnut intervention (Figure 1).

\section{RESULTS}

\subsection{Effects of Walnut Feeding on Body Weight and} Relative Organ Weight and Biochemical Parameters. Long-term fructose-fed animals tended to eat less and, expectedly, drink more, in comparison to fructose-naive animals and irrespectively of walnut kernels. ${ }^{24}$ The feeding behavior is graphically summarized in Figure $S 1$ and absolute organ weights in Table S1. Both fructose and walnut consumption tended to induce an increase in body weight (Figure 2A). These treatments also significantly increased final body weight $(p<$ 0.05 for the main effects) (Figure 2B) and reduced relative kidney weight, while walnut feeding significantly increased relative adipose tissue weight, with no changes in relative liver weight (Figure 2C).

Fructose induced increases in serum triglycerides (TAG), total cholesterol (TC), low-density lipoprotein cholesterol (LDL-c), and high-density lipoprotein cholesterol (HDL-c), confirming the metabolic syndrome model, while the walnut consumption reduced HDL-c and tended to reduce TC, in both healthy animals and animals at metabolic risk, without affecting the TC/HDL-c ratio (Table 1 ). Only for the aspartate aminotransferase (AST), the decreasing effect of walnuts tended to appear in fructose-fed animals ( $p$ for interaction $=0.069$ ). Otherwise, we observed no interaction between the walnuts and fructose consumption on the anthropometry and biochemical parameters of the treated animals.

2.2. Effects of Walnut Feeding on Tissue-Specific n-6/ n-3 Ratio. Irrespectively of the metabolic health, walnuts induced up to a 2 -fold decrease in the cumulative $n-6 / n-3$ content of the total lipid pool in plasma, liver, adipose tissue, and kidney $(p<0.05$, Table 2$)$.

Across the investigated tissues, the most abundant fatty acids were PA (range: $18-33 \%$ of the total lipid pool) and LA (range: $10-41 \%$ of the total lipid pool). In epididymal adipose tissue solely, the oleic acid (C18:1 n-9) content was prominent (range: $23-30 \%$ of the total lipid pool), while plasma, liver, and kidney also presented with substantial portions of AA (range: $17-33 \%$ of the total lipid pool).

We further analyzed correlations between serum lipids as proxies of metabolic disturbance and $n-6 / n-3$ ratio across the tissues and depending on the treatment (Table S2). Only in fructose-fed animals, not treated with walnuts (the F group), we observed a strong positive correlation between $\mathrm{TC}$ and the ratio in plasma $(r=0.84, p=0.004)$, and a tendency in the liver $(r=$ $0.63, p=0.069)$ and adipose tissue $(r=0.66, p=0.051)$. In fructose-naive animals on walnut feeding, the positive correlation was present in kidney tissue only (Table S2).

2.3. Effects of Walnut Feeding on Endogenous Metabolic Conversion toward Long-Chain Fatty Acids. We analyzed the effects of walnut consumption on fatty acid metabolic conversion toward longer-chain fatty acid products, as seen in the following ratios: POA/PA, AA/LA, EPA/ $\alpha \mathrm{LNA}$, and DHA $/ \alpha$ LNA (Figure 3).

In plasma and liver total lipids, walnuts decreased the AA/LA ratio and tended to decrease POA/PA levels ( $p$ for interaction < 0.05; except for liver POA/PA, $p$ for interaction $=0.086$ ). Within the same tissues, consumption of walnuts markedly affected the 
A

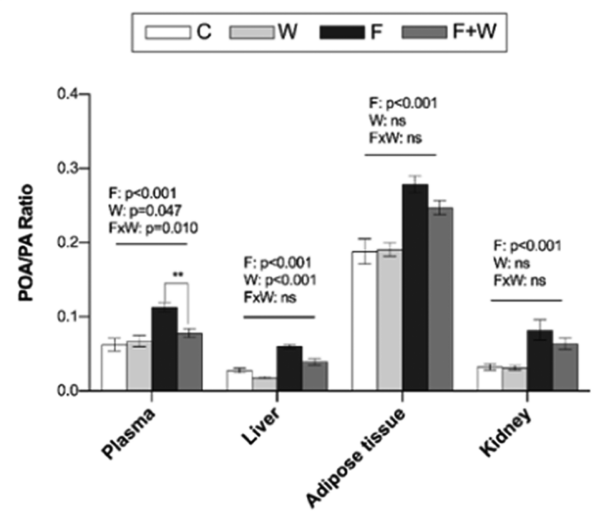

C

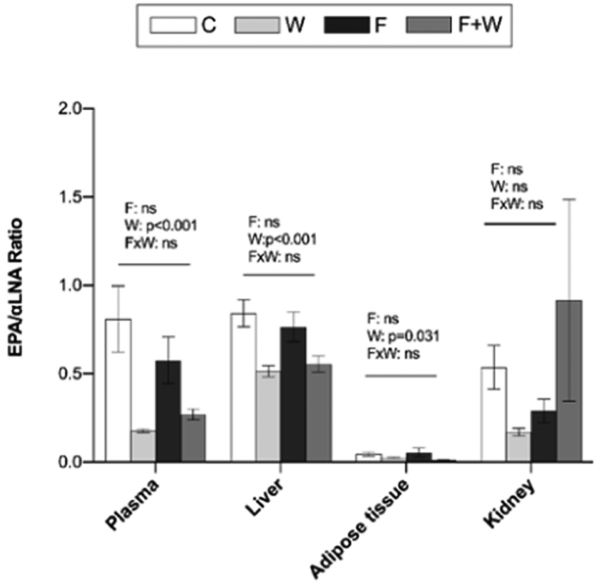

B

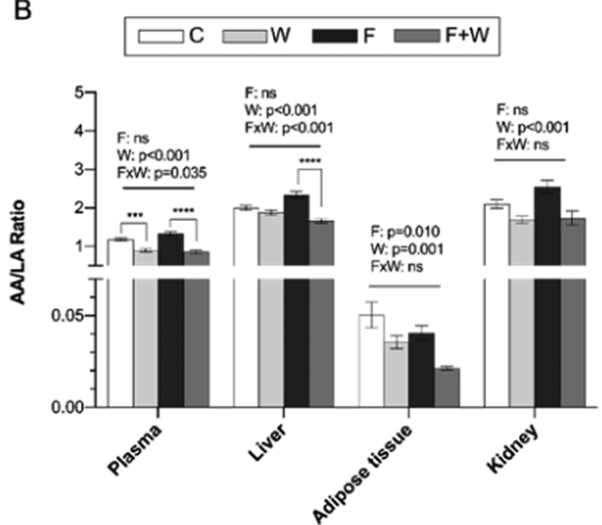

D

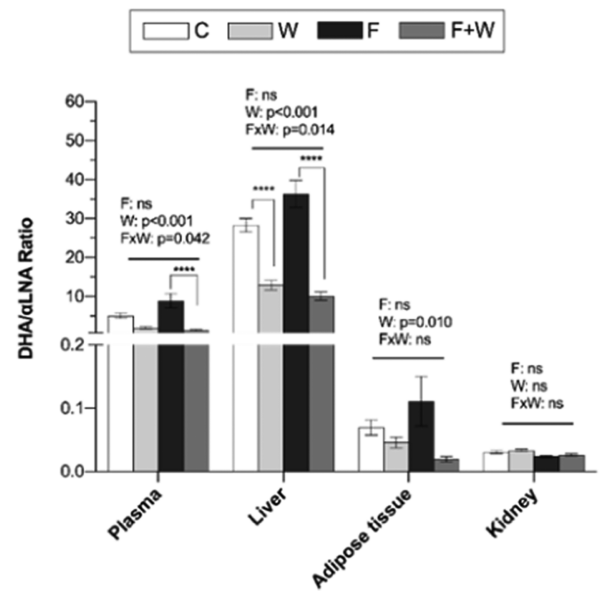

Figure 3. Effects of walnut feeding on tissue-specific fatty acid metabolic conversion indices. (A) PA/POA, (B) AA/LA, (C) EPA/ $\alpha$ LNA, and (D) $\mathrm{DHA} / \alpha$ LNA ratios within the total lipid pools in plasma, liver, epididymal tissue, and kidney of control or fructose-fed rats with or without walnut feeding. Results are analyzed by two-way ANOVA, followed by Sidak's post hoc test. Data are presented as mean \pm SEM, $n=7-9$, ** $p<0.01$, *** $p<$ $0.001, * * * * p<0.0001$. C-control, $\mathrm{W}$-walnuts, $\mathrm{F}$-fructose, $\mathrm{F}+\mathrm{W}$-fructose plus walnuts.

DHA/ $\alpha$ LNA portion, with up to a 4-fold decrease in the animals at metabolic risk. All of the effects were more prominent in animals at metabolic risk.

Walnuts decreased AA/LA, EPA/ $\alpha$ LNA, and DHA/ $\alpha$ LNA in adipose tissue, and AA/LA in the kidney, yet the effects were not dependent on the level of the metabolic risk ( $p$ for interaction $>$ $0.05)$.

2.4. Effects of Walnut Feeding on Tissue-Specific Distribution of Fatty Acids in Rats at Metabolic Risk. In animals at metabolic risk induced by high fructose intake, consumption of walnuts affected the tissue-specific distribution of fatty acids within the total lipid pools (Figure 4).

In plasma, the walnut consumption significantly decreased POA, VA, and DHA contents but increased the portion of LA and EPA (Figure 4). Walnuts increased LA and DHA content in rat liver (Figure 4B,C). In epididymal adipose tissue, the walnut consumption increased stearic acid and decreased VA (Figure $4 \mathrm{~A})$ and tended to decrease long-chain $n-3$ docosapentaenoic acid $(p$ for interaction $=0.062)$ and DHA $(p$ for interaction $=$ 0.068) (Figure 4C). In the kidney, the walnut treatment decreased VA, while tended to decrease OA ( $p$ for interaction $=$ 0.065 ) and increase LA and EPA content ( $p$ for interaction $=$ 0.061 and 0.059 ) (Figure 4).

2.5. Tissue-Specific Walnut Effects Independent of Fructose-Induced Metabolic Risk. Independently of the metabolic risk imposed by the fructose treatment, walnuts modulated individual tissue-specific fatty acid contents of the total lipid pool. Across all of the investigated tissues, walnuts induced up to a 3 -fold increase in $\alpha$ LNA and concomitantly decreased AA. Walnut consumption decreased PA levels, and in all tissues except adipose, increased docosapentaenoic and decreased adrenic acid content. The OA content was decreased upon walnut consumption in all tissues except for kidney. Walnuts elevated LA in adipose tissue and kidney. Tissuespecific walnut effects on individual fatty acid content, independent on metabolic risk, are presented in Figure 4 (W factor).

\section{DISCUSSION}

Our results demonstrate the effects of consumption of walnut kernels for 6 weeks on the lowering of the n-6/n-3 ratio in plasma, liver, epididymal adipose tissue, and kidney, in both healthy animals and high-fructose-fed animals at metabolic risk. The high n-6/n-3 ratio is associated with obesity and metabolic syndrome $^{5}$ and cardiovascular disease, ${ }^{25}$ and our results highlight the promising aspect of walnuts consumption for cardiometabolic benefits. The metabolic risk in our study was induced by preceding fructose consumption, and although we proved no interaction among fructose and walnut consumption toward the $n-6 / n-3$ ratio, in our study, the lowest values for the ratio were found in fructose-naive animals upon 6 weeks of walnut consumption. Thus, our results also add value to walnuts consumption in the primary prevention setting. 
A

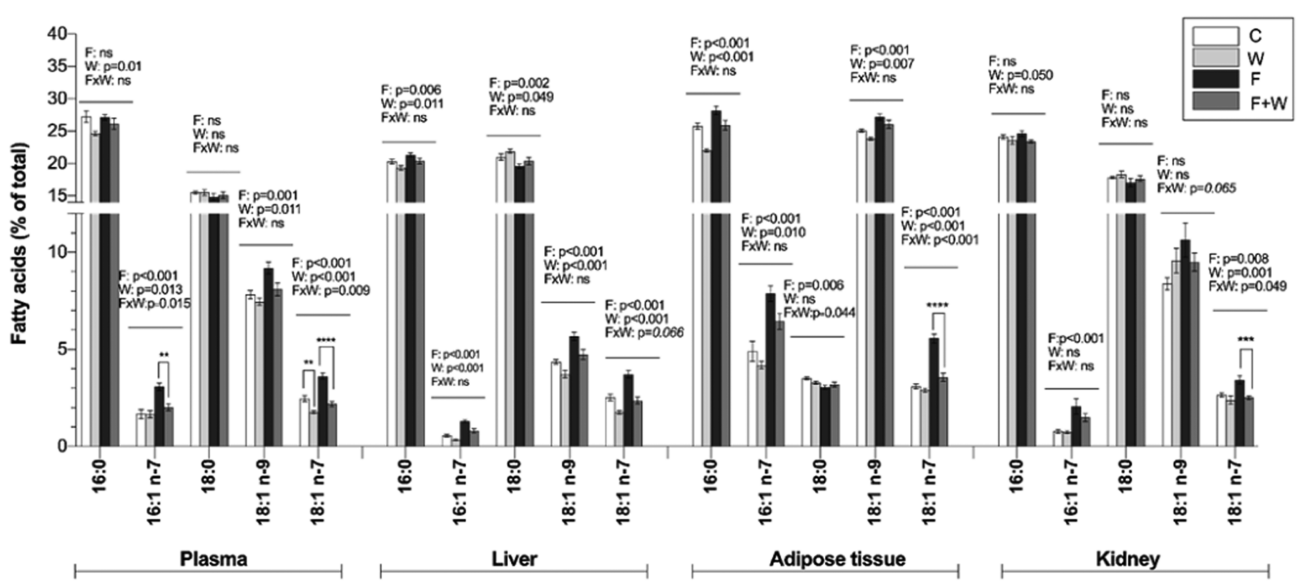

B
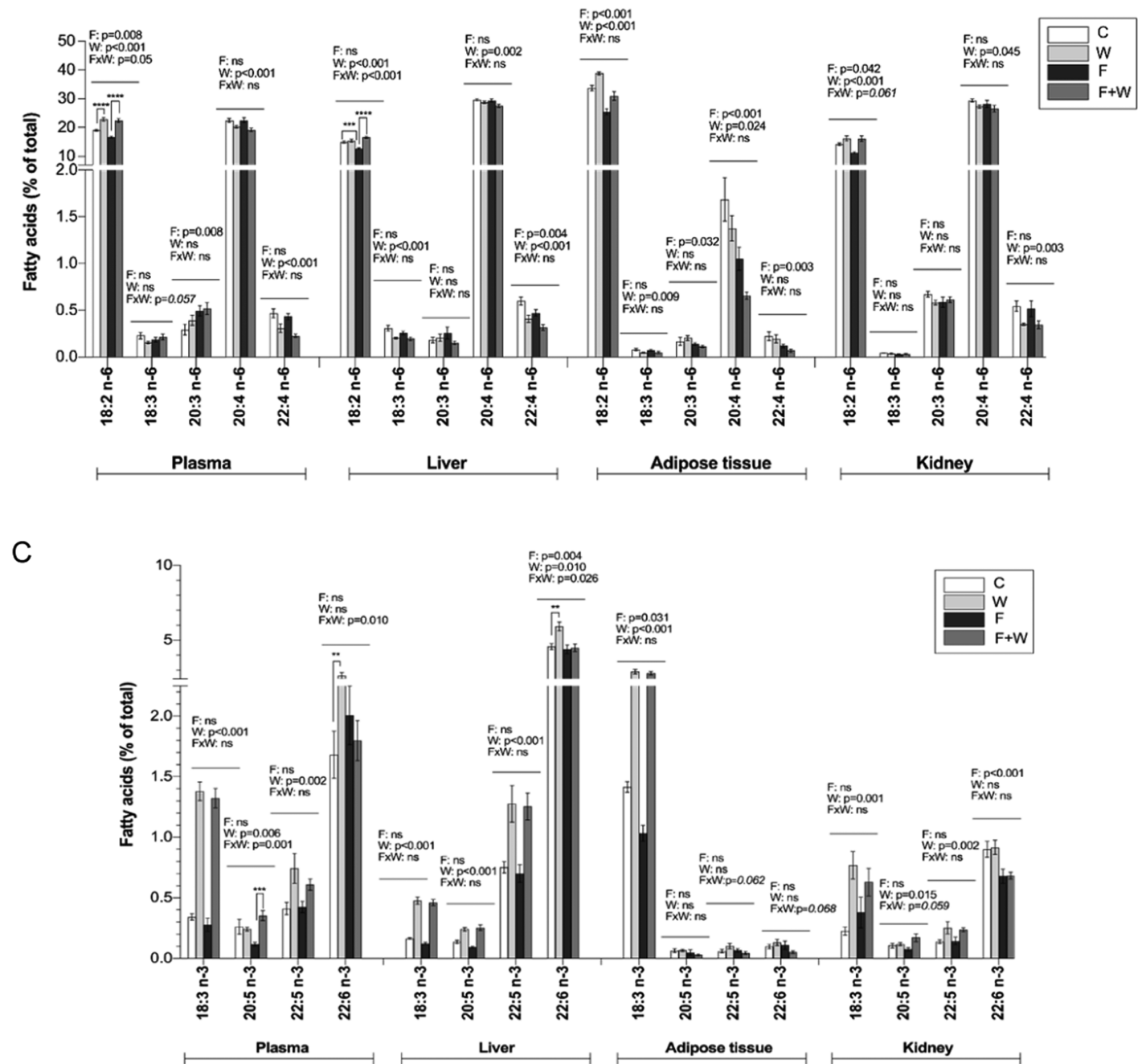

Figure 4. Impact of walnut consumption on tissue-specific distribution of fatty acids. The relative percentage of the individual fatty acids belonging to (A) de novo lipogenesis, (B) n-6, and (C) n-3 pathway across plasma, liver, epididymal adipose tissue, and kidney total lipids in fructose-naive or fructose-fed male Wistar rats upon 6-week dietary intake of walnuts kernels. Results are analyzed by two-way ANOVA, followed by Sidak's post hoc test. Data are presented as mean $\pm \mathrm{SEM}, n=7-9, * * p<0.01, * * * p<0.001, * * * * p<0.0001$. C—control, W-walnuts, F-fructose, $\mathrm{F}+\mathrm{W}-$ fructose plus walnuts.

Previously, a varying dietary $n-6 / n-3$ ratio based on the soybean and cod liver oil was directly associated with the corresponding ratio in plasma, adipose tissue, and liver in pregnant Sprague Dawley rats. ${ }^{26}$ Furthermore, a study in Sprague Dawley male rats demonstrated the effects of cottonseed oil in comparison to corn oil consumption on increasing saturated-to-monounsaturated fatty acid ratio in serum, liver, and adipose tissue, with, however, lowering and no effects toward n-3 and n- 6 tissue fractions, respectively. ${ }^{27}$ The authors concluded that further strategies are needed to modulate 
n-6/n-3 tissue levels for desirable health effects, and our study supports the role of dietary walnuts for the ratio optimization.

To the best of our knowledge, no previous study investigated metabolic breakdown of the fats contained in walnuts, and herein, we report the comprehensive distribution of fatty acids across the plasma, adipose tissue, liver, and kidney upon walnut consumption in metabolically healthy and unhealthy animals. In addition to the salient importance of the $n-6 / n-3$ ratio, individual fatty acids hold promising potential in cardiovascular disease risk optimization. ${ }^{28}$ We thus investigated the effects of walnut consumption on individual fatty acids and corresponding metabolic ratios and showed that in animals at metabolic risk, walnuts decreased plasma levels of POA $/ \mathrm{PA}$ ( $p$ for interaction $=$ $0.010)$. The ratio is a surrogate marker of stearoyl-CoA desaturase activity, involved in DNL, and directly associated with MetS. ${ }^{3}$ Dietary n-3 might inhibit the DNL pathway by suppressing malonyl-CoA conversion to fatty acids, ${ }^{29}$ the mechanism potentially underlying the POA/PA lowering effects observed in our study. Although fructose induced POA/PA increase in other tissues as well, we observed no reversing effects of walnuts in tissues other than plasma, contrasting previous findings in Sprague Dawley rats showing that n-3 fatty acids reversed the cyclosporine-induced decrease in stearoyl-CoA desaturase activity in the liver and kidney. ${ }^{30}$ However, unlike our study that used dietary supplementation with n-3-rich walnuts, this study assessed the impact of an n-3 ethyl ester concentrate (EPA and DHA), administrated by gavage at a $300 \mathrm{mg} / \mathrm{kg} /$ day dose, thus making the results difficult to compare due to differing study designs. Irrespective of fructose feeding, walnuts in our study, however, decreased the POA/PA liver ratio, pointing out alleviating effects on fat synthesis performed in the liver upon walnut consumption. Accordingly, long-term walnut consumption in high-fat-fed mice was shown to alleviate liver steatosis and adipose tissue inflammation. ${ }^{17}$ Deregulated liver fatty acid profile, in terms of depleted long-chain $n-3$ pools coupled with disrupted inflammatory homeostasis, might be associated with liver inflammation and chronic liver condition, ${ }^{31,32}$ and our results propel the importance of the walnut feeding for liver health.

In addition, regardless of the metabolic health of the rats, walnuts decreased the AA/LA and $\mathrm{DHA} / \alpha \mathrm{LNA}$ ratios, particularly in plasma and liver total lipids pool, mostly due to the increase in individual LA and $\alpha$ LNA contents. Walnut consumption increased $\alpha$ LNA content across all investigated tissues and irrespective of metabolic burden, while the increase in LA content was more prominent in animals at metabolic risk, specifically in plasma, liver, and kidney. The changes in essential fatty acid levels of tissues reflect the compositional characteristics of study walnuts but might also mirror the metabolic competition. Namely, in the presence of even low $\alpha$ LNA levels, the LA conversion is mitigated due to the extensive utilization of $\alpha \mathrm{LNA}$ as a competitive substrate for the enzymes. $\alpha \mathrm{LNA}$-rich walnuts thus might have induced a competitive substrate utilization in the n-3 fatty acid conversion pathway in our study, also in line with the herein observed decrease in AA and adrenic acid levels, as the n-6 metabolic products. We also observed that walnuts increased n-3 metabolic products across the tissues, including EPA and docosapentaenoic acid. From the inflammation standpoint, the findings outline favorable effects of the consumption, as AA is speculated for the proinflammatory ${ }^{33}$ and EPA for the opposite effects, both commonly ascribed to the balance of the metabolic products, i.e., eicosanoids. ${ }^{8}$ Notably, dietary $\alpha$ LNA is with higher conversion capacity toward blood
EPA than DHA, while the DHA content is largely determined by preformed dietary DHA, ${ }^{34}$ which might underpin the nonsignificant changes in the tissue DHA observed in the study herein.

In line with the results from previous studies, fructose feeding in our study induced metabolic disturbances, as manifested in increased serum TAG, TC, and LDL-c levels. ${ }^{35,36}$ Independently of metabolic risk, walnuts reduced serum HDL-c, while not significantly affecting TAG, TC, and LDL-c, contrasting previous research on cholesterol-lowering effects of dietary walnuts, particularly in hyperlipidemic subjects. ${ }^{37-39}$ Previously, walnut supplementation reduced TC as well as HDL-c, the latter in line with our findings. ${ }^{40}$ A recent meta-analysis in 1059 subjects showed that the walnut-enriched diet reduced TAG, $\mathrm{TC}$, and LDL-c, without affecting HDL-c and weight change. ${ }^{41}$ On the other hand, the results from a case-control study in subjects with MetS demonstrated modest effects of nuts, including walnut consumption toward LDL-c and HDL-c levels. ${ }^{42}$ It should be noted that unlike humans, rodents are characterized by a circulating HDL-dominant lipoprotein profile and lack a cholesteryl ester transfer protein that transfers cholesteryl ester from HDL-c to apoB-containing lipoproteins. ${ }^{43}$ Therefore, precautions should be taken when comparing data from human studies with those from rodents due to the differences in lipoprotein physiology. Nonetheless, the results from animal feeding studies using walnuts also remain inconclusive. These discrepancies in the observed effects could be explained by the high variability in study designs in terms of duration and dose of supplementation, and model of metabolic disturbances. For example, a previous study on male albino rats demonstrated superior effects of a 10-week walnut oil supplementation ( $31.7 \%$ in the diet) in comparison to other plant oils, in terms of TAG-decreasing potential. ${ }^{44}$ In the same study, the increase in $\alpha$ LNA content within the experimental diet alleviated the TC increase. ${ }^{44}$ On the contrary, a study in mice with genetically determined MetS showed that a diet supplemented with $0.3 \%$ ground walnuts for 21 weeks induced an increase in TC and TAG, followed by the upregulation of hepatic profibrotic and proinflammatory genes. ${ }^{45}$ Further on, in high-fat-fed C57BL/6J mice, supplementation of a diet with $14.2 \%$ of ground walnuts inversely affected TAG liver accumulation, with no influence on animal body weight or liver weight upon $6^{18}$ and 20 weeks. ${ }^{17}$ The 6 -week study, although comparable to ours in terms of treatment dose and duration, was however designed to assess the preventive potential of the 6-week walnut intake on early signaling events in nonalcoholic fatty liver disease, ${ }^{18}$ while our study aimed at investigating the healing potential of dietary walnuts when a metabolic burden is already present. In addition, TC levels in our study directly correlated with the n-6/n-3 levels in plasma, liver, and adipose tissue only in the fructose-fed animals, eating no walnuts, which was not observed in walnut feeding groups, indicating the healing potential of the dietary walnuts in the state of metabolic disturbances. Notably, walnut treatment in our study induced increase in body weight and relative epididymal adipose tissue weight, which was virtually higher in fructose-fed animals. Clinical studies demonstrated that although energydense, habitual consumption of walnuts for 6 months was associated with neither weight gain nor effects on anthropometric parameters. ${ }^{46}$ As reported before, the selected daily amount of walnuts in our study conferred an excessive energy intake, ${ }^{24}$ possibly linked with the unfavorable changes in anthropometry indices. Another study showed that mixed nut 
and pistachio consumption is associated with improved serum lipid profile in atherogenic diet-fed rats, with no changes in weight. ${ }^{47}$ In the latter study, the groups were, however, conformed with initial body weight, while we randomly selected the animals, possibly causing the unfavorable body weight changes. The FDA health claim recommends a daily amount of $43 \mathrm{~g}$ of dietary nuts as safe and beneficial for human consumption; $^{48}$ the question, however, remains on the daily walnut amount to be incorporated in dietary patterns for safe consumption in terms of avoiding excessive caloric intake and elevated adiposity yet pursuing favorable cardiometabolic effects.

We observed no changes in glucose parameters, similarly to that reported before. ${ }^{17}$ Furthermore, we showed a tendency toward the AST-protective effect in fructose-fed rats, indicating the hepatoprotective effects of dietary walnuts in metabolic conditions. The hepatoprotective effects of dietary walnuts were also reported in the liver of mice fed a high-fat diet, mediated through the reversal of SIRT1, AMPK, and FAS function and modulation of the expression of different genes involved in lipid metabolism. ${ }^{18,19}$ A study on male Wistar rats showed the antioxidative and hepatoprotective potential of walnut consumption against ethanol-induced oxidative disbalance, through the prevention of lipid peroxidation. ${ }^{16}$ Herein, we showed that 6-week walnut consumption particularly affected metabolic ratios of fatty acids and serum lipid profile, in favor of omega-3 products and cumulative content, that might be underpinned by nutrigenomic effects of $\alpha$ LNA-rich walnuts, through modulation of transcription factors such as PPAR and LXR. ${ }^{49}$ The limitation of our study is the lack of information on the regulatory and mechanistic background of the herein observed effects, and the field necessitates further research.

Aside from $\alpha \mathrm{LNA}$, walnuts contain monounsaturated fatty acids, LA, fibers, and are rich sources of minerals such as magnesium, calcium, arginine, and phytochemicals like polyphenols, which all could exert beneficial effects and might potentially act synergistically. ${ }^{6}$ Among 1113 food items examined, walnuts marked the second place with regard to the content of antioxidant compounds. ${ }^{50}$ However, whole walnuts rich in $\alpha \mathrm{LNA}$ are indicated as a candidate saturated fat replacement food for favorable cardiometabolic benefits, ${ }^{51}$ and we suggest that the $\alpha$ LNA contained in the walnuts triggers the herein observed decline in the $n-6 / n-3$ ratio.

In our study, adding walnut kernels in the experimental consumption did not match for the polyunsaturated fatty acid content within the standard consumption provided. The reason for this lies in the fact that we aimed to simulate the addition of a handful of walnuts as a healthy snack within the habitual human diet. In addition, the walnut treatment duration was not concomitant with fructose treatment, to confirm the effects of walnuts in alleviating already established metabolic risk and further confirm its dietary potential in the healthcare of metabolic syndrome. The strength of our study lies in the comprehensive assessment of the habitual walnut consumption on full fatty acid profiles and indicators of metabolic conversion across various animal tissues, which, to the best of our knowledge, has not been reported previously. We also included the kidney as previously it has been reported that high fructose intake induced DNL in both liver and kidney in rats. ${ }^{52}$ To the best of our knowledge, previous studies scarcely addressed kidney-specific fatty acids profiles. Given the fructose-induced hypertriglyceridemia, future studies should explore the effects of walnuts on fatty acid fractions across the tissues.
In conclusion, irrespective of metabolic burden induced by high fructose consumption, the addition of walnut kernels into daily feeding lowered tissue omega-6/omega-3 ratio in Wistar rats upon consumption for 6 weeks. Further mechanistic studies should explain the background of the observed effects, and nutritional studies in humans should focus on the safe daily amount of walnuts to avoid excessive caloric intake, still pertaining to their health-promoting properties.

\section{METHODS}

4.1. Walnut Characterization. Walnuts (Juglans regia) used in this study were obtained from a local market. The complete fatty acid, macronutrient, and mineral contents of the walnuts are described elsewhere. ${ }^{9}$ The most abundant fatty acids in walnuts were LA with $63.2 \%$, oleic acid (C18:1 n-9) with $14.5 \%$, and $\alpha$ LNA, with $11.2 \%$ total fatty acids identified by gas chromatography analysis. ${ }^{9}$ Due to the PUFA susceptibility to oxidation when provided as part of the standard animal consumption, ${ }^{53}$ we distributed the whole walnut kernels to the experimental animals and strictly followed to ensure every animal ate its portion of the daily walnut amount at the moment of the delivery.

4.2. Study Animals and Experimental Design. Thirty-six male Wistar rats, obtained from the vivarium of the Institute of Nuclear Sciences "Vinča", Serbia, were used in this study. Animals were housed in groups of three per cage at controlled room temperature $\left(22 \pm 1^{\circ} \mathrm{C}\right)$ and humidity, under a $12 \mathrm{~h}$ light/ dark cycle and free access to water and standard rat chow (Veterinarski Zavod, Subotica, Serbia) (Table S3) to allow acclimatization. Fructose was purchased from Omnia Nisasta Sanayi ve Ticaret A.S. (Adana, Turkey).

At the age of 21 days, the rats were randomly divided into two groups: (1) control group (C) with unlimited access to standard diet and drinking water and (2) fructose group $(\mathrm{F})$, provided ad libitum with standard diet and $10 \%(\mathrm{w} / \mathrm{v})$ fructose solution in drinking water (Figure 1), previously demonstrated to induce metabolic disturbances in male Wistar rats. ${ }^{35}$ After 9 weeks, every group was additionally randomized to (3) walnuts group (W) that received a standard diet enriched with walnuts and drinking water, and (4) fructose plus walnuts group $(F+W)$ provided with $10 \%$ fructose solution and walnut-enriched diet. In $\mathrm{W}$ and $\mathrm{F}+\mathrm{W}$ groups, animals were fed walnut kernels $(2.4 \mathrm{~g} /$ animal/day) in addition to a standard diet for the next 6 weeks (Figure 1). The $2.4 \mathrm{~g}$ corresponded to one kernel half of a larger walnut, which was provided per animal. The animals would always eat a dose of walnuts in the presence of the experimenters within a maximum of $5 \mathrm{~min}$, leaving no doubt whether the consumption was even and whether there were unconsumed nuts left in the cage. The selected amount of walnuts resembles a $56 \mathrm{~g} /$ day dose in humans, previously shown to exert relevant cardiometabolic effects. ${ }^{13,41,46}$ The human equivalent dose (HED) was calculated according to the method by ReaganShaw and co-workers that takes into account between-species metabolic differences, where (HED in $\mathrm{mg} / \mathrm{kg}$ ) = animal dose $(\mathrm{mg} / \mathrm{kg}) \times$ animal $K_{\mathrm{m}}(6$ for rats $) \div$ Human $K_{\mathrm{m}}(37)$, for a $60 \mathrm{~kg}$ human. ${ }^{54}$ The animals were given free access to water and standard diets during the course of the experiments. Standard food and liquid consumption were recorded daily. Energy intake was calculated as a sum of $\mathrm{kJ}$ from the standard food, fructose solution, and walnut kernels. Body mass was recorded weekly. All animal procedures were approved by the Ethical Committee for the Use of Laboratory Animals of the Institute of Nuclear 
Sciences Vinča and performed following the guidelines of Directive 2010/63/EU of the European Parliament.

4.3. Collection of Animal Tissues. Similar to our previous work, ${ }^{35}$ at the end of the experiment, the animals were sacrificed by decapitation without anesthesia, to avoid potential interference with parameters of insulin sensitivity and sugar metabolism. ${ }^{55,56}$ Blood was collected into the EDTA tubes and centrifuged at $2000 \mathrm{rpm}$ for $10 \mathrm{~min}$ to obtain plasma samples. Biochemical parameters were measured using a Cobas c111 clinical chemistry analyzer (Roche Diagnostics, Basel, Switzerland) and Roche's diagnostic kits following the manufacturer's instructions, and glucose was measured by a personal analyzer Accu Check Active from the same manufacturer. Liver, epididymal fat pads, and kidneys were removed, then rinsed with ice-cold sodium chloride $(0.154 \mathrm{~mol} / \mathrm{L})$, weighed, and stored at $-80{ }^{\circ} \mathrm{C}$ until the tissue-specific fatty acid composition was determined.

4.4. Fatty Acid Analysis in Total Lipids. Total lipids were extracted from liver, adipose tissue, kidney, and plasma by the Folch method $^{57}$ using chloroform/methanol $(2: 1, \mathrm{v} / \mathrm{v})$ with $0.05 \%(\mathrm{w} / \mathrm{v})$ butylated hydroxytoluene.

Fatty acids were esterified to fatty acid methyl esters with $3 \mathrm{M}$ hydrochloric acid in methanol with $2 \%(\mathrm{w} / \mathrm{v})$ butylated hydroxytoluene at $85{ }^{\circ} \mathrm{C}$ for $1 \mathrm{~h}$. Samples were brought to ambient temperature, mixed with hexane, and centrifuged at $3000 \mathrm{rpm}$ for $10 \mathrm{~min}$. The upper phase was collected and dried under a gentle stream of $\mathrm{N}_{2}$. The samples were reconstituted in hexane before analysis. Fatty acid methyl esters were separated by a Shimadzu GC-2014 gas chromatograph (Kyoto, Japan) equipped with a flame ionization detector and an RTX 2330 column (RESTEK, Bellefonte, PA) that was $60 \mathrm{~m}$ long with an internal diameter of $0.25 \mathrm{~mm}$ and film thickness of $0.2 \mu \mathrm{m}$. The injector and detector temperatures were set at 220 and $250{ }^{\circ} \mathrm{C}$, respectively. The column temperature settings were as follows: $100{ }^{\circ} \mathrm{C}$ for $5 \mathrm{~min}$, increased to $175^{\circ} \mathrm{C}$ at the rate of $10^{\circ} \mathrm{C} / \mathrm{min}$; $175^{\circ} \mathrm{C}$ for $27 \mathrm{~min}$, increased to $220^{\circ} \mathrm{C}$ at the rate of $3{ }^{\circ} \mathrm{C} / \mathrm{min}$; and $220^{\circ} \mathrm{C}$ for $35 \mathrm{~min}$. Fatty acids were identified by comparing retention times with PUFA-2 standard mixture and Supelco 37 Component Fame Mix (Supelco, Inc., Bellefonte, PA), and the content of the individual fatty acid in the investigated tissues was expressed as a percentage of the total identified fatty acids in total lipid pool within a tissue. For the purposes of the current study, we identified the following fatty acids: PA, POA, stearic acid, vaccenic acid (C18:1 n-7), oleic acid, LA, $\gamma$-linolenic acid (C18:3 n-6), dihomo- $\gamma$-linolenic acid (C20:3 n-6), AA, adrenic acid (C22:4n-6), $\alpha$ LNA, EPA, docosapentaenoic acid (C22:5n3 ), and DHA. Fatty acid product-to-precursor ratios were used to estimate the effects of walnuts on the activities of enzymes involved in the synthesis of long-chain fatty acid products. Thus, $\mathrm{AA} / \mathrm{LA}$ ratio was used as an estimate of the overall desaturation and elongation activity in the n- 6 pathway, and EPA $/ \alpha$ LNA and $\mathrm{DHA} / \alpha \mathrm{LNA}$ ratios as the respective activities in the $\mathrm{n}-3$ pathway. To tackle the effects on DNL, we also evaluated treatment effects on POA/PA as the DNL surrogate marker.

4.5. Statistical Analyses. Data are presented as mean \pm SEM. Interindividual treatment effects were analyzed by twoway ANOVA, including fructose and walnut treatment as independent factors. The interaction term was accounted for in the statistical models. Data were analyzed by SPSS software, version 24.0 (IBM Corp., Armonk, NY), and $p<0.05$ was considered significant. As the primary outcome of the study was to explore the effects of walnuts against fructose intervention on tissue-specific fatty acid distribution, the results are discussed accordingly when the interaction showed statistical significance. Otherwise, the main walnut treatment effects are discussed independently. For the significant interactions, we applied Sidak's post hoc test for pairwise comparisons for each level of fructose treatment, i.e., to compare walnut effects independently in fructose-naive vs. fructose-fed animals. All graphical representations were created using GraphPad Prism for Macintosh, version 8.1.1 (San Diego, CA).

\section{ASSOCIATED CONTENT}

\section{Supporting Information}

The Supporting Information is available free of charge at https://pubs.acs.org/doi/10.1021/acsomega.0c03784.

Absolute organ weights (Table S1), correlations between serum lipids and $n-6 / n-3$ ratio across the tissues (Table S2), composition of a standard diet (Table S3), and feeding behavior parameters (Figure S1) (PDF)

\section{AUTHOR INFORMATION}

\section{Corresponding Author}

Manja M. Zec - Centre of Excellence in Nutrition and Metabolism Research, Institute for Medical Research, National Institute of Republic of Serbia, University of Belgrade, Belgrade 11000, Serbia; Email: manjazecimr@gmail.com

\section{Authors}

Irena Krga - Centre of Excellence in Nutrition and Metabolism Research, Institute for Medical Research, National Institute of Republic of Serbia, University of Belgrade, Belgrade 11000, Serbia; 이이.orid.org/0000-0002-2073-2896

Marija Takić - Centre of Excellence in Nutrition and Metabolism Research, Institute for Medical Research, National Institute of Republic of Serbia, University of Belgrade, Belgrade 11000, Serbia

Jasmina Debeljak-Martačic - Centre of Excellence in Nutrition and Metabolism Research, Institute for Medical Research, National Institute of Republic of Serbia, University of Belgrade, Belgrade 11000, Serbia

Goran Koricanac - Laboratory for Molecular Biology and Endocrinology, Vinča Institute of Nuclear Sciences, National Institute of Republic of Serbia, University of Belgrade, Belgrade 11001, Serbia

Slavica Rankovic - Centre of Excellence in Nutrition and Metabolism Research, Institute for Medical Research, National Institute of Republic of Serbia, University of Belgrade, Belgrade 11000, Serbia

Tamara Popovic - Centre of Excellence in Nutrition and Metabolism Research, Institute for Medical Research, National Institute of Republic of Serbia, University of Belgrade, Belgrade 11000, Serbia

Marija Pantelić - Laboratory for Molecular Biology and Endocrinology, Vinca Institute of Nuclear Sciences, National Institute of Republic of Serbia, University of Belgrade, Belgrade 11001, Serbia

Maria Glibetic - Centre of Excellence in Nutrition and Metabolism Research, Institute for Medical Research, National Institute of Republic of Serbia, University of Belgrade, Belgrade 11000, Serbia

Complete contact information is available at:

https://pubs.acs.org/10.1021/acsomega.0c03784 


\section{Author Contributions}

${ }^{\S}$ M.M.Z. and I.K. contributed equally to this work.

\section{Funding}

This work was supported by the Ministry of Education, Science and Technological Development of the Republic of Serbia, under the contract numbers 451-03-68/2020-14/200015 and 451-03-68/2020-14/200017.

\section{Notes}

The authors declare no competing financial interest.

\section{ACKNOWLEDGMENTS}

The authors are thankful to the technical personnel involved in the experimental manipulations.

\section{ABBREVIATIONS}

$\alpha$ LNA, $\alpha$-linolenic acid; AA, arachidonic acid; ALT, alanine aminotransferase; AST, aspartate aminotransferase; C, control group; DHA, docosahexaenoic acid; DNL, de novo lipogenesis; EPA, eicosapentaenoic acid; F, fructose group; F + W, fructose plus walnuts group; HDL-c, high-density lipoprotein cholesterol; LA, linoleic acid; LDL-c, low-density lipoprotein cholesterol; n-3, omega-3 fatty acids; n-6, omega-6 fatty acids; MetS, metabolic syndrome; PA, palmitic acid; POA, palmitoleic acid; TAG, triglycerides; TC, total cholesterol; W, walnuts group

\section{REFERENCES}

(1) Grundy, S. M.; Becker, D.; Clark, L. T.; Cooper, R. S.; Denke, M. A.; Howard, J.; Hunninghake, D. B.; Illingworth, D. R.; Luepker, R. V.; McBride, P.; et al. Detection, Evaluation, and Treatment of High Blood Cholesterol in Adults (Adult Treatment Panel III). Circulation 2002, $17,3143-3421$

(2) Julibert, A.; Bibiloni, M.; Bouzas, C.; Martínez-González, M.; Salas-Salvadó, J.; Corella, D.; Zomeño, M.; Romaguera, D.; Vioque, J.; Alonso-Gómez, A.; et al. Total and Subtypes of Dietary Fat Intake and Its Association with Components of the Metabolic Syndrome in a Mediterranean Population at High Cardiovascular Risk. Nutrients 2019, 11, 1493.

(3) Mayneris-Perxachs, J.; Guerendiain, M.; Castellote, A. I.; Estruch, R.; Covas, M. I.; Fitó, M.; Salas-Salvadó, J.; Martínez-González, M. A.; Aros, F.; Lamuela-Raventós, R. M.; et al. Plasma Fatty Acid Composition, Estimated Desaturase Activities, and Their Relation with the Metabolic Syndrome in a Population at High Risk of Cardiovascular Disease. Clin. Nutr. 2014, 33, 90-97.

(4) Chilton, F.; Murphy, R.; Wilson, B.; Sergeant, S.; Ainsworth, H.; Seeds, M.; Mathias, R. Diet-Gene Interactions and PUFA Metabolism: A Potential Contributor to Health Disparities and Human Diseases. Nutrients 2014, 6, 1993-2022.

(5) Simopoulos, A. An Increase in the Omega-6/Omega-3 Fatty Acid Ratio Increases the Risk for Obesity. Nutrients 2016, 8, 128.

(6) Zec, M.; Glibetic, M. Health Benefits of Nut Consumption. In Reference Module in Food Science; Elsevier, 2018; pp 1-13.

(7) Bashan, I.; Bakman, M. The Effect of Daily Walnut Consumption on Dyslipidemia. J. Food Qual. 2018, 2018, 1-6.

(8) Serhan, C. N. Pro-Resolving Lipid Mediators Are Leads for Resolution Physiology. Nature 2014, 510, 92-101.

(9) Petrović-Oggiano, G.; Debeljak-Martačić, J.; Ranković, S.; Pokimica, B.; Miríc, A.; Glibetić, M.; Popović, T. The Effect of Walnut Consumption on N-3 Fatty Acid Profile of Healthy People Living in a Non-Mediterranean West Balkan Country, a Small Scale Randomized Study. Nutrients 2020, 12, 192.

(10) Chong, M. F. F.; Hodson, L.; Bickerton, A. S.; Roberts, R.; Neville, M.; Karpe, F.; Frayn, K. N.; Fielding, B. A. Parallel Activation of de Novo Lipogenesis and Stearoyl-CoA Desaturase Activity after $3 \mathrm{~d}$ of High-Carbohydrate Feeding. Am. J. Clin. Nutr. 2008, 87, 817-823.

(11) Wu, J. H. Y.; Lemaitre, R. N.; Imamura, F.; King, I. B.; Song, X.; Spiegelman, D.; Siscovick, D. S.; Mozaffarian, D. Fatty Acids in the de
Novo Lipogenesis Pathway and Risk of Coronary Heart Disease: The Cardiovascular Health Study. Am. J. Clin. Nutr. 2011, 94, 431-438.

(12) Guasch-Ferré, M.; Liu, X.; Malik, V. S.; Sun, Q.; Willett, W. C.; Manson, J. E.; Rexrode, K. M.; Li, Y.; Hu, F. B.; Bhupathiraju, S. N. Nut Consumption and Risk of Cardiovascular Disease. J. Am. Coll. Cardiol. 2017, 70, 2519-2532.

(13) Xiao, Y.; Huang, W.; Peng, C.; Zhang, J.; Wong, C.; Kim, J. H.; Yeoh, E.; Su, X. Effect of Nut Consumption on Vascular Endothelial Function: A Systematic Review and Meta-Analysis of Randomized Controlled Trials. Clin. Nutr. 2018, 37, 831-839.

(14) Beulen, Y.; Martínez-González, M.; van de Rest, O.; SalasSalvadó, J.; Sorlí, J.; Gómez-Gracia, E.; Fiol, M.; Estruch, R.; SantosLozano, J.; Schröder, H.; et al. Quality of Dietary Fat Intake and Body Weight and Obesity in a Mediterranean Population: Secondary Analyses within the PREDIMED Trial. Nutrients 2018, 10, 2011.

(15) Sun, Y.; Qi, G.; Li, D.; Meng, H.; Zhu, Z.; Zhao, Y.; Qi, Y.; Zhang, X. Walnut (Juglans regia L.) Kernel Extracts Protect Against Isoproterenol-Induced Myocardial Infarction in Rats. Rejuvenation Res. 2019, 22, 306-312.

(16) Bati, B.; Celik, I.; Dogan, A. Determination of Hepatoprotective and Antioxidant Role of Walnuts Against Ethanol-Induced Oxidative Stress in Rats. Cell Biochem. Biophys. 2015, 71, 1191-1198.

(17) Choi, Y.; Abdelmegeed, M. A.; Akbar, M.; Song, B.-J. Dietary Walnut Reduces Hepatic Triglyceride Content in High-Fat-Fed Mice via Modulation of Hepatic Fatty Acid Metabolism and Adipose Tissue Inflammation. J. Nutr. Biochem. 2016, 30, 116-125.

(18) Choi, Y.; Abdelmegeed, M. A.; Song, B.-J. Preventive Effects of Dietary Walnuts on High-Fat-Induced Hepatic Fat Accumulation, Oxidative Stress and Apoptosis in Mice. J. Nutr. Biochem. 2016, 38, 7080.

(19) Luo, T.; Miranda-Garcia, O.; Adamson, A.; Hamilton-Reeves, J.; Sullivan, D. K.; Kinchen, J. M.; Shay, N. F. Consumption of Walnuts in Combination with Other Whole Foods Produces Physiologic, Metabolic, and Gene Expression Changes in Obese C57BL/6J HighFat-Fed Male Mice. J. Nutr. 2016, 146, 1641-1650.

(20) Fink, A.; Rüfer, C. E.; Le Grandois, J.; Roth, A.; Aoude-Werner, D.; Marchioni, E.; Bub, A.; Barth, S. W. Dietary Walnut Oil Modulates Liver Steatosis in the Obese Zucker Rat. Eur. J. Nutr. 2014, 53, 645660.

(21) Gencoglu, H.; Orhan, C.; Tuzcu, M.; Sahin, N.; Juturu, V.; Sahin, K. Effects of Walnut Oil on Metabolic Profile and Transcription Factors in Rats Fed High-Carbohydrate-/-Fat Diets. J. Food Biochem. 2020, 44, No. e13235.

(22) Song, L.; Zhou, H.; Yu, W.; Ding, X.; Yang, L.; Wu, J.; Song, C. Effects of Phytosterol Ester on the Fatty Acid Profiles in Rats with Nonalcoholic Fatty Liver Disease. J. Med. Food 2020, 23, 161-172.

(23) Koletzko, B.; Reischl, E.; Tanjung, C.; Gonzalez-Casanova, I.; Ramakrishnan, U.; Meldrum, S.; Simmer, K.; Heinrich, J.; Demmelmair, H. FADS1 and FADS2 Polymorphisms Modulate Fatty Acid Metabolism and Dietary Impact on Health. Annu. Rev. Nutr. 2019, $39,21-44$.

(24) Stanisic, J.; Ivkovic, T.; Romic, S.; Zec, M.; Culafic, T.; Stojiljkovic, M.; Koricanac, G. Beneficial Effect of Walnuts on Vascular Tone Is Associated with Akt Signalling, Voltage-Dependent Calcium Channel LTCC and ATP-Sensitive Potassium Channel Kv1.2. Int. J. Food Sci. Nutr. 2020, 1-11.

(25) Simopoulos, A. P. New Products from the Agri-Food Industry: The Return of n-3 Fatty Acids into the Food Supply. Lipids 1999, 34, S297-S301.

(26) Kassem, A. A.; Abu Bakar, M. Z.; Yong Meng, G.; Mustapha, N. M. Dietary (n-6: N-3) Fatty Acids Alter Plasma and Tissue Fatty Acid Composition in Pregnant Sprague Dawley Rats. Sci. World J. 2012, 2012, 1-7.

(27) Radcliffe, J. D.; Czajka-Narnis, D. M.; Imrhan, V. Fatty Acid Composition of Serum, Adipose Tissue, and Liver in Rats Fed Diets Containing Corn Oil or Cottonseed Oil. Plant Foods Hum. Nutr. 2004, $59,73-77$. 
(28) Jackson, K. H.; Harris, W. S. Blood Fatty Acid Profiles: New Biomarkers for Cardiometabolic Disease Risk. Curr. Atheroscler. Rep. 2018, 20, 22.

(29) Hellerstein, M. K. De Novo Lipogenesis in Humans: Metabolic and Regulatory Aspects. Eur. J. Clin. Nutr. 1999, 53, s53-s65.

(30) Lee, S. M.; Lee, M. H.; Son, Y. K.; Kim, S. E.; Park, Y.; Rha, S. H.; An, W. S. Omega-3 Fatty Acid Decreases Oleic Acid by Decreasing SCD-1 Expression in the Liver and Kidney of a Cyclosporine-Induced Nephropathy Rat Model. Renal Failure 2019, 41, 211-219.

(31) Boyer-Diaz, Z.; Morata, P.; Aristu-Zabalza, P.; Gibert-Ramos, A.; Bosch, J.; Gracia-Sancho, J. Oxidative Stress in Chronic Liver Disease and Portal Hypertension: Potential of DHA as Nutraceutical. Nutrients 2020, 2627.

(32) Boyer-Diaz, Z.; Domingo, J. C.; de Gregorio, E.; Manicardi, N.; Aristu-Zabalza, P.; Cordobilla, B.; Abad-Jordà, L.; Ortega-Ribera, M.; Fernández-Iglesias, A.; Marí, M.; et al. A Nutraceutical Rich in Docosahexaenoic Acid Improves Portal Hypertension in a Preclinical Model of Advanced Chronic Liver Disease. Nutrients 2019, 11, 2358.

(33) Sonnweber, T.; Pizzini, A.; Nairz, M.; Weiss, G.; Tancevski, I. Arachidonic Acid Metabolites in Cardiovascular and Metabolic Diseases. Int. J. Mol. Sci. 2018, 3285.

(34) Brenna, J. T.; Salem, N.; Sinclair, A. J.; Cunnane, S. C. $\alpha$ Linolenic Acid Supplementation and Conversion to n-3 Long-Chain Polyunsaturated Fatty Acids in Humans. Prostaglandins, Leukotrienes Essent. Fatty Acids 2009, 80, 85-91.

(35) Stanišić, J.; Korićanac, G.; Culafić, T.; Romić, S.; Stojiljković, M.; Kostić, M.; Pantelić, M.; Tepavčević, S. Low Intensity Exercise Prevents Disturbances in Rat Cardiac Insulin Signaling and Endothelial Nitric Oxide Synthase Induced by High Fructose Diet. Mol. Cell. Endocrinol. 2016, 420, 97-104.

(36) Cardinali, D. P.; Scacchi Bernasconi, P. A.; Reynoso, R.; Reyes Toso, C. F.; Scacchi, P. Melatonin May Curtail the Metabolic Syndrome: Studies on Initial and Fully Established Fructose-Induced Metabolic Syndrome in Rats. Int. J. Mol. Sci. 2013, 14, 2502-2514.

(37) Almario, R. U.; Vonghavaravat, V.; Wong, R.; Kasim-Karakas, S. E. Effects of Walnut Consumption on Plasma Fatty Acids and Lipoproteins in Combined Hyperlipidemia. Am. J. Clin. Nutr. 2001, 74, 72-79.

(38) Ros, E.; Núñez, I.; Pérez-Heras, A.; Serra, M.; Gilabert, R.; Casals, E.; Deulofeu, R. A Walnut Diet Improves Endothelial Function in Hypercholesterolemic Subjects: A Randomized Crossover Trial. Circulation 2004, 109, 1609-1614.

(39) Zambón, D.; Sabaté, J.; Muñoz, S.; Campero, B.; Casals, E.; Merlos, M.; Laguna, J. C.; Ros, E. Substituting Walnuts for Monounsaturated Fat Improves the Serum Lipid Profile of Hypercholesterolemic Men and Women. A Randomized Crossover Trial. Ann. Intern. Med. 2000, 132, 538-546.

(40) Sabate, J.; Fraser, G. E.; Burke, K.; Knutsen, S. F.; Bennett, H.; Lindsted, K. D. Effects of Walnuts on Serum Lipid Levels and Blood Pressure in Normal Men. N. Engl. J. Med. 1993, 328, 603-607.

(41) Guasch-Ferré, M.; Li, J.; Hu, F. B.; Salas-Salvadó, J.; Tobias, D. K. Effects of Walnut Consumption on Blood Lipids and Other Cardiovascular Risk Factors: An Updated Meta-Analysis and Systematic Review of Controlled Trials. Am. J. Clin. Nutr. 2018, 108, 174-187.

(42) Casas-Agustench, P.; López-Uriarte, P.; Bulló, M.; Ros, E.; Cabré-Vila, J. J.; Salas-Salvadó, J. Effects of One Serving of Mixed Nuts on Serum Lipids, Insulin Resistance and Inflammatory Markers in Patients with the Metabolic Syndrome. Nutr. Metab. Cardiovasc. Dis. 2011, 21, 126-135.

(43) Yin, W.; Carballo-Jane, E.; McLaren, D. G.; Mendoza, V. H.; Gagen, K.; Geoghagen, N. S.; McNamara, L. A.; Gorski, J. N.; Eiermann, G. J.; Petrov, A.; et al. Plasma Lipid Profiling across Species for the Identification of Optimal Animal Models of Human Dyslipidemia. J. Lipid Res. 2012, 53, 51-65.

(44) Hammad, K. S. M.; Hallabo, S. A.; Galal, S. M. Protective Effect of Linseed Oil and Walnuts against Hypercholesterolemia Induced by Atherogenic Diet in Rats. J. Food Nutr. Res. 2015, 3, 653-660.

(45) Scott, N.; Ellmers, L.; Pilbrow, A.; Thomsen, L.; Richards, A.; Frampton, C.; Cameron, V. Metabolic and Blood Pressure Effects of
Walnut Supplementation in a Mouse Model of the Metabolic Syndrome. Nutrients 2017, 9, 722.

(46) Njike, V. Y.; Ayettey, R.; Petraro, P.; Treu, J. A.; Katz, D. L. Walnut Ingestion in Adults at Risk for Diabetes: Effects on Body Composition, Diet Quality, and Cardiac Risk Measures. BMJ Open Diabetes Res. Care 2015, 3, No. e000115.

(47) Hong, M.; Groven, S.; Marx, A.; Rasmussen, C.; Beidler, J. AntiInflammatory, Antioxidant, and Hypolipidemic Effects of Mixed Nuts in Atherogenic Diet-Fed Rats. Molecules 2018, 23, 3126.

(48) FDA Food; Administration, D. Nuts and Coronary Heart Disease (Docket No 02P-0505). Qualified Health Claims: Letters of Enforcement Discretion; US Food and Drug Administration: Rockville, 2003; pp 1-4.

(49) Li, A. C.; Glass, C. K. PPAR- and LXR-Dependent Pathways Controlling Lipid Metabolism and the Development of Atherosclerosis. J. Lipid Res. 2004, 45, 2161-2173.

(50) Halvorsen, B. L.; Carlsen, M. H.; Phillips, K. M.; Bøhn, S. K.; Holte, K.; Jacobs, D. R.; Blomhoff, R. Content of Redox-Active Compounds (Ie, Antioxidants) in Foods Consumed in the United States. Am. J. Clin. Nutr. 2006, 84, 95-135.

(51) Tindall, A. M.; Petersen, K. S.; Skulas-Ray, A. C.; Richter, C. K.; Proctor, D. N.; Kris-Etherton, P. M. Replacing Saturated Fat With Walnuts or Vegetable Oils Improves Central Blood Pressure and Serum Lipids in Adults at Risk for Cardiovascular Disease: A Randomized Controlled-Feeding Trial. J. Am. Heart Assoc. 2019, 8, No. 69.

(52) Milutinović, D. V.; Brkljačić, J.; Teofilović, A.; Bursać, B.; Nikolić, M.; Gligorovska, L.; Kovačević, S.; Djordjevic, A.; Preitner, F.; Tappy, L.; et al. Chronic Stress Potentiates High Fructose-Induced Lipogenesis in Rat Liver and Kidney. Mol. Nutr. Food Res. 2020, 64, No. 1901141.

(53) Zhang, J.; Freund, M. A.; Culler, M. D.; Yang, R.; Chen, P. B.; Park, Y.; Decker, E. A.; Zhang, G. How To Stabilize $\omega-3$ Polyunsaturated Fatty Acids (PUFAs) in an Animal Feeding Study?-Effects of the Temperature, Oxygen Level, and Antioxidant on Oxidative Stability of $\omega-3$ PUFAs in a Mouse Diet. J. Agric. Food Chem. 2020, No. 8298.

(54) Reagan-Shaw, S.; Nihal, M.; Ahmad, N. Dose Translation from Animal to Human Studies Revisited. FASEB J. 2008, 22, 659-661.

(55) De Oliveira, J. C.; Ludemann Camargo, R.; Barella, L. F.; Chaves Souto Branco, R.; Gravena, C.; Grassiolli, S.; Torrezan, R.; Cezar De Freitas Mathias, P. Anesthetic-Induced Transient Hyperglycemia and Insulin Resistance Do Not Depend on the Sympathoadrenal Axis. Minerva Endocrinol. 2013, 38, 379-388.

(56) Saha, J. K.; Xia, J.; Grondin, J. M.; Engle, S. K.; Jakubowski, J. A. Acute Hyperglycemia Induced by Ketamine/Xylazine Anesthesia in Rats: Mechanisms and Implications for Preclinical Models. Exp. Biol. Med. 2005, 230, 777-784.

(57) Folch, J.; Lees, M.; Solane Stanley, G. H. A Simple Method for the Isolation and Purification of Total Lipides from Animal Tissues. J. Biol. Chem. 1957, 226, 497-509. 